\section{Políticas de saúde e concepções de surdez e de deficiência auditiva no SUS: um monólogo?}

\author{
Health policies and definitions of deafness and \\ hearing impairment in the SUS: a monologue?
}

\section{Políticas de salud y concepciones de sordera y deficiencia auditiva en el SUS: ¿un monólogo?}

Carla Soleman 1,2

Aylene Bousquat 1

\section{Resumo}

Duas concepções teóricas são centrais na discussão sobre a deficiência auditiva e a surdez: a orgânico-biológica e a socioantropológica. Elas são fundamentalmente distintas na definição de uma hipotética fronteira da normalidade e no entendimento dos surdos como uma minoria linguística. O objetivo deste trabalho foi o de identificar como essas concepções se expressaram nas politicas de saúde voltadas às pessoas com deficiência auditiva/surdez no Sistema Unico de Saúde (SUS). Foi realizada um pesquisa documental com foco nas políticas de saúde voltadas à deficiência auditiva/surdez de 1990 a 2019. Os documentos $(n=185)$ foram identificados na Biblioteca Virtual em Saúde e no Sistema de Legislação da Saúde. Desses, foram selecionados 11 que versavam sobre normativas de implementação tecnológica ou ação prática na assistência com foco na deficiência auditiva/surdez, os demais tinham caráter fundamentalmente pontual. Os resultados evidenciam que todas as politicas analisadas foram pautadas na concepção orgânico-biológica, pois nenhuma referência às particularidades linguísticas e culturais do surdo foi identificada. Essa lógica pode contribuir para dificultar o acesso dessa população ao sistema de saúde, especialmente ao aumentar a barreira comunicacional. Para que de fato se cumpram os princípios doutrinários do SUS é central que as políticas de saúde incorporem os aspectos socioculturais, refletindo em suas formulações o surdo como um cidadão que apreende o mundo pelas vivências e experiências prioritariamente visuais, no qual a língua de sinais é elemento central.

Pessoas com Deficiência Auditiva; Surdez; Linguas de Sinais; Politica de Saúde

\author{
Correspondência \\ C. Soleman \\ Faculdade Saúde Pública, Universidade de São Paulo. \\ Av. Dr. Arnaldo 715, São Paulo, SP 01246-904, Brasil. \\ carlasoleman@usp.br \\ 1 Faculdade de Saúde Pública, Universidade de São Paulo, São \\ Paulo, Brasil. \\ 2 Secretaria de Saúde, Prefeitura Municipal de São José do Rio \\ Preto, São José do Rio Preto, Brasil.
}




\section{Introdução}

A deficiência auditiva e a surdez têm sido tratadas como sinônimos em boa parte da produção científica no campo da saúde. Parte-se de uma visão dicotômica entre normalidade e anormalidade, na qual a incapacidade em detectar, discriminar e processar os sons do ambiente e da fala é uma anormalidade a ser corrigida, ou seja, as pessoas surdas são vistas com base na deficiência auditiva e, consequentemente, necessitam restaurar a sua capacidade de ouvir 1,2,3. Essa redução da surdez a um déficit auditivo expressa a concepção orgânico-biológica da surdez 4,5 .

Por outro lado, a História da educação de surdos evidencia o desenvolvimento de uma habilidade de comunicação que independe da funcionalidade do aparelho auditivo, pois usa a via visuo-gestual para a comunicação por meio de sinais, capacidade esta que vem possibilitando conquistas de direitos sociais das pessoas surdas desde o século XVIII e é traço central na concepção socioantropológica da surdez. Esse olhar enfatiza outras formas de vivenciar as diferenças na qual os surdos são um grupo que se identifica como uma minoria linguística que valoriza sua história, propõe uma pedagogia própria para a educação das crianças surdas e não se considera deficiente 5 .

A discussão de fundo que separa as duas concepções é a definição de uma hipotética fronteira da normalidade, problemática seminalmente abordada por Canguilhem 6 (p. 71) ao afirmar que "a fronteira entre o normal e o patológico é imprecisa para diversos indivíduos considerados simultaneamente, mas é perfeitamente precisa para um único e mesmo indivíduo considerado sucessivamente", ou seja, a experiência do homem na relação da sua condição com o meio é que determina a normatividade desta condição. No caso da surdez, a privação sensorial é vivenciada de diferentes formas pelos indivíduos, a depender do quanto esta condição modifica ou não sua relação com o meio.

Vale ressaltar que a partir da década de 1960, por meio dos chamados estudos culturais, a cultura e a linguagem, ou as formações discursivas presentes na sociedade, passaram a ter papel de destaque na teoria do processo de construção cultural das identidades 7 . No caso das comunidades surdas, vários autores do campo da educação identificaram a existência de diversas comunidades surdas com representações e identidades diversificadas sobre a surdez na sociedade 1,8,9. Tais comunidades são formadas não só por surdos, mas por todos que interagem com eles, portanto, pais, amigos, professores que conhecem e usam a língua de sinais para se comunicar 10.

A língua brasileira de sinais (Libras) é reconhecida como meio legal de comunicação e expressão das pessoas surdas 11 , e o decreto que a regulamenta define pessoa surda como aquela que, por ter perda auditiva, compreende e interage com o mundo por meio de experiências visuais, manifestando sua cultura principalmente pelo uso da libras. Já a deficiência auditiva seria a perda bilateral, parcial ou total, de quarenta e um decibéis (dB) ou mais, aferida por audiograma ${ }^{12}$. Nesse complexo universo da deficiência auditiva encontram-se tanto as pessoas oralizadas, tendo como primeira língua o português, e aquelas não oralizadas, que majoritariamente têm a Libras como primeira língua 13.

Concepções distintas implicam ações e condutas também diferentes. No caso da elaboração, formulação, implementação e avaliação das políticas de saúde essa discussão deveria ser enfrentada, pois estima-se que no mundo 466 milhões de pessoas têm deficiência auditiva 14. As iniquidades em saúde são muito mais prevalentes em grupos minoritários, tais como os surdos, uma vez que as reais necessidades de saúde destas pessoas são pouco estudadas e mal atendidas 15.

No Brasil, em 2013, estimou-se que 1,1\% da população possuía deficiência auditiva, sendo que $0,9 \%$ adquiriu esta deficiência por doença ou acidente e $0,2 \%$ a possuía desde o nascimento. Entre as pessoas com deficiência auditiva, 20,6\% apresentaram grau intenso ou muito intenso de limitações, não conseguindo realizar atividades habituais 16 , o que corresponde em números absolutos a 1.799.885 pessoas com um maior comprometimento do sistema auditivo. De acordo com uma pesquisa realizada mundialmente, no Brasil existiriam 1.700.000 surdos usuários de língua de sinais ${ }^{17}$, o que coincide com o número de pessoas que referem apresentar grande dificuldade em ouvir, e salienta a importância de considerar as diferentes concepções para a construção das políticas públicas de saúde voltadas às pessoas nesta condição.

Apesar da magnitude dessa população, análises das políticas de saúde voltadas para essas pessoas ainda são extremamente incomuns no Brasil, especialmente aquelas que tentam incorporar em sua lógica o entendimento de que essa parcela da população constitui uma minoria linguística 3,8,17,18. 
Dentro desse cenário, o objetivo deste artigo é identificar as concepções de surdez expressas nas políticas públicas de saúde voltadas às pessoas com deficiência auditiva no âmbito do Sistema Único de Saúde (SUS), no período de 1990 até 2019.

\section{Referencial teórico}

As duas concepções de surdez consideradas neste estudo são a orgânico-biológica, pautada na medicina e na habilitação ou reabilitação do aparelho auditivo, aprendizagem das habilidades auditivas e o desenvolvimento de uma linguagem auditivo-oral com vistas à comunicação por meio da oralidade e da fala, e a concepção socioantropológica, que considera a particularidade linguística e cultural da surdez, valorizando o desenvolvimento da linguagem visuo-gestual, a comunicação por meio da língua de sinais e o bilinguismo $1,2,3,4,5$.

Na perspectiva orgânico-biológica, a surdez é vista como uma inabilidade do indivíduo diante de estímulos sonoros, uma privação sensorial causada por diferentes fatores, em qualquer fase do processo do desenvolvimento humano ${ }^{19}$. Nessa concepção acredita-se que, a partir da reabilitação do déficit e do treino das habilidades auditivas, todos os indivíduos surdos tenham condição para o desenvolvimento da língua oral. Sustenta a ideia de que o desenvolvimento cognitivo é dependente da eficácia oral, estabelecendo uma relação equivocada entre linguagem e língua oral 4 . Tal modelo afirma que a língua de sinais não constitui um verdadeiro sistema linguístico e que a mesma impede ou limita o desenvolvimento da linguagem oral 20.

Uma das questões centrais para o entendimento da concepção socioantropológica é que esta está ancorada no referencial da cultura surda, ou seja, considera as diferentes formas de apreensão das informações e experimentação do mundo. Em geral, o termo cultura nos remete a um conjunto de práticas simbólicas de um determinado grupo. A cultura surda faz referência ao uso da língua de sinais, às estratégias sociais e aos mecanismos compensatórios que os surdos realizam para agir no mundo, tais como o despertador que vibra, a campainha que aciona a luz, o uso de mensagens visuais e de texto, a educação bilíngue etc. ${ }^{9}$. Reconhecer a cultura surda é reconhecer as variadas especificidades manifestadas na língua, nos hábitos, nos modos de socialização e de funcionamento cognitivo que dão origem a uma identidade própria. Não se trata de incentivar a criação de grupos minoritários à margem da sociedade, mas sim considerar a diferenciação linguística como necessária para possibilitar o desenvolvimento normal da cognição, da subjetividade, da expressividade e da cidadania da pessoa surda 21. Considerando que nos grupos de surdos também há ouvintes que usam a língua de sinais, implica a possibilidade de uma interação social em relação de igualdade, que aproxima os surdos de outras culturas, e que difere dos ouvintes em função dos processos comunicativos e não pela alteração orgânica funcional 3 .

Conferir à língua de sinais o estatuto de língua não tem apenas repercussões linguísticas, mas também repercussões sociais, pois, se ser normal implica ter língua, e se a anormalidade é a ausência de língua e de tudo o que ela representa, ao se configurar a língua de sinais como língua do surdo o estatuto do que é normal também muda. A língua de sinais acaba por oferecer uma possibilidade de legitimação do surdo como sujeito de linguagem, transformando a "anormalidade" em diferença. A constituição da identidade pelo surdo não está necessariamente relacionada à língua de sinais, mas sim à presença de um meio de existir no mundo como "falante", de constituir sua própria subjetividade pela linguagem e às implicações disto nas suas relações sociais 9 .

\section{Percurso metodológico}

Com base nesse referencial foi realizada uma pesquisa documental, na qual foram analisadas as políticas públicas de saúde com enfoque na deficiência auditiva/surdez, vigentes no SUS de 1990 a 2019.

A pesquisa documental é um procedimento que utiliza métodos e técnicas para a apreensão, compreensão e análise de documentos dos mais variados tipos. Possibilita ampliar o entendimento de objetos cuja compreensão necessita de contextualização histórica e sociocultural, permite acrescentar 
a dimensão tempo à compreensão do social, favorecendo a observação do processo de maturação ou de evolução de grupos, conceitos, conhecimentos, comportamentos e práticas 22.

Os documentos foram identificados na Biblioteca Virtual em Saúde (BVS) e no Sistema de Legislação da Saúde (SAUDELEGIS), utilizando-se os descritores: surdez, perda auditiva, pessoas com deficiência auditiva, audiologia, saúde auditiva, aparelho auditivo, implante coclear. Essa busca identificou 185 documentos, que foram analisados em relação aos objetivos de cada normatização. Normatizações de cunho apenas organizacional/burocrático, tais como alteração nos valores ou códigos da tabela SUS para procedimentos já existentes, habilitação de serviços de referência, distribuição de teto financeiro entre entes federados e serviços de referência e similares foram excluídas. Foram identificados 11 documentos que expressavam mudanças nas normas de implementação tecnológica ou ação prática de assistência com foco na deficiência auditiva/surdez dentro do SUS.

Os documentos foram analisados buscando-se identificar como as diferentes concepções de surdez são expressas nessas políticas voltadas às pessoas com deficiência auditiva/surdez, com base na identificação dos principais elementos que caracterizam as diferentes concepções (Figura 1).

\section{Resultados}

O cuidado à saúde da pessoa com deficiência é assegurado como direito pela Constituição Federal de 1988, que dispõe sobre o apoio às "pessoas portadoras de deficiência", atribuindo ao setor saúde a promoção de ações preventivas, a criação de uma rede de serviços especializados em reabilitação e habilitação, a garantia de acesso aos estabelecimentos de saúde e do adequado tratamento no seu interior, além do desenvolvimento de programas de saúde voltados para estas pessoas e desenvolvidos com a participação da sociedade 23 .

Com o objetivo de promover a redução da incidência de deficiência no país e garantir a atenção integral, o Ministério da Saúde instituiu o Programa de Atenção à Saúde da Pessoa Portadora de Deficiência, e em decorrência foi editado um conjunto de portarias que estabeleceram normas e incluíram os procedimentos de reabilitação em nível ambulatorial e hospitalar no SUS, regulamentando, inclusive, a concessão de órteses e próteses 23 .

\section{Figura 1}

Concepções de surdez.
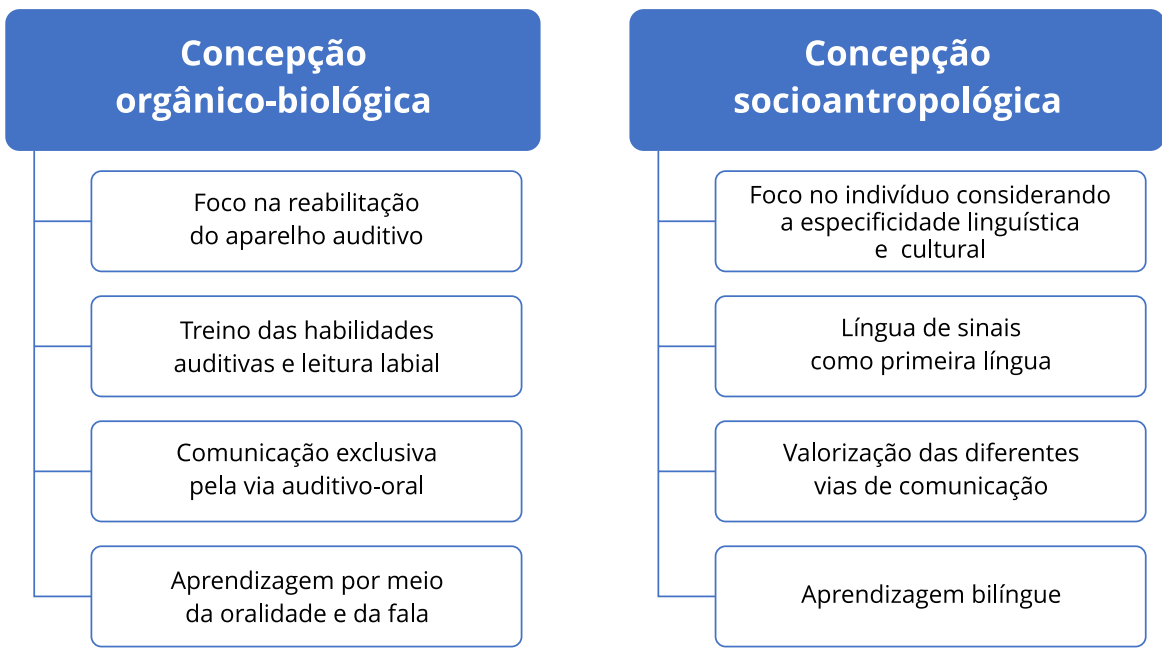

Fonte: elaborado pelas autoras. 
A partir da linha do tempo, apresentada na Figura 2, visualiza-se que o SUS incorporou a oferta de recursos para o tratamento da deficiência auditiva/surdez a partir de 1993, visando principalmente à garantia de financiamento e à inclusão de procedimentos específicos para a assistência, cujo objetivo é a reabilitação da função auditiva por meio da concessão de aparelhos de amplificação sonora individual (AASI) e cirurgia de implante coclear 24,25.

As duas portarias que seguem as pioneiras, no ano de 1999 26,27, apenas regulamentam os critérios para a prescrição das ações clínico-terapêuticas anteriormente implementadas, mantendo a concepção orgânico-biológica como centro da política pública.

Em 2000, as ações ampliaram por meio da regulamentação, no âmbito ambulatorial, do diagnóstico, adaptação e acompanhamento de indivíduos deficientes auditivos, com base em uma reestruturação dos procedimentos e serviços das tabelas SUS 28. Houve direcionamento de recursos financeiros para o diagnóstico e a concessão de AASI em detrimento da reabilitação auditiva, uma vez que procedimentos como a terapia fonoaudiológica e as ações de saúde auditiva na atenção básica eram realizados esporadicamente 29 . Vale salientar que, apesar de ampliadas as ações para o cuidado em deficiência auditiva/surdez, todos os procedimentos implementados se referem a procedimentos diagnósticos e de reabilitação da função auditiva.

Em 2004, com base na necessidade do Ministério da Saúde em rever a efetividade dos procedimentos de concessão de AASI 29, foi instituída a Política Nacional de Atenção à Saúde Auditiva (PNASA), uma ação mais densa, com um olhar mais ampliado para a integralidade das ações em saúde auditiva, uma vez que trazia em seu escopo o desenvolvimento de estratégias de promoção, prevenção, tratamento e reabilitação em todos os níveis de atenção 30 . A assistência foi organizada em Redes Estaduais de Atenção à Saúde Auditiva, constituída com base nos componentes atenção básica, média e alta complexidade, e determinando ações que deveriam ser realizadas de forma interdisciplinar nos diferentes níveis 31 .

\section{Figura 2}

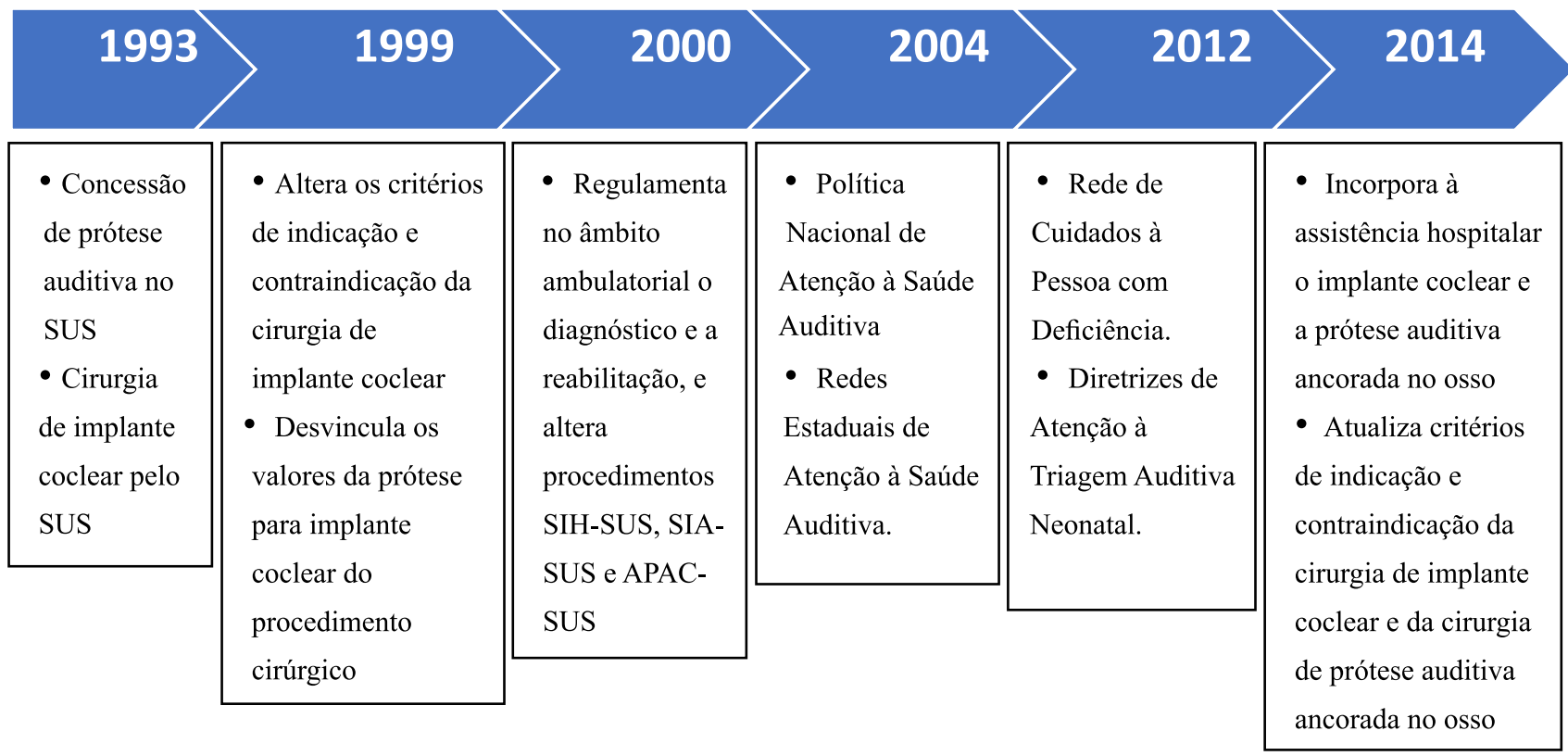

APAC: autorização de procedimentos de alta complexidade; SIA: Sistema de Informações Ambulatoriais; SIH: Sistema de Informações Hospitalares. Fonte: elaborado pelas autoras. 
A Rede de Cuidados à Pessoa com Deficiência (RCPD), política pública implementada em 2012 e vigente atualmente, visa a ampliar o acesso e qualificar o atendimento às pessoas com deficiência no SUS, com foco na organização de rede e na atenção integral à saúde. Traz como diretrizes: garantia de autonomia, independência e liberdade às pessoas com deficiência para fazerem as próprias escolhas, promoção da equidade e do respeito às diferenças e aceitação de pessoas com deficiência, com enfrentamento de estigmas e preconceitos, garantia de acesso e de qualidade dos serviços, mas em relação à deficiência auditiva/surdez também se apresenta como uma política limitada às ações de reabilitação do aparelho auditivo, pois não menciona aspectos relacionados à cultura surda 32 .

Em 2012, o Ministério da Saúde aprovou as Diretrizes de Atenção à Triagem Auditiva Neonatal, que tem por finalidade a identificação precoce da deficiência auditiva nos neonatos, com o objetivo de encaminhá-los para o diagnóstico e posteriores intervenções, considerando que o diagnóstico funcional e a intervenção iniciados antes dos seis meses de vida da criança possibilitam, em geral, melhores resultados para o desenvolvimento da função auditiva, da linguagem, da fala, do processo de aprendizagem e, consequentemente, a inclusão no mercado de trabalho e melhor qualidade de vida 33.

As últimas políticas implementadas no Brasil com foco na deficiência auditiva/surdez datam de 2014 e também são pautadas na concepção orgânico-biológica, pois ampliam a oferta de tecnologias para a reabilitação da função auditiva no SUS. Aprovam diretrizes gerais, ampliam e incorporam procedimentos para a atenção especializada, atualizando os critérios de indicação e contraindicação da cirurgia de implante coclear, e estabelecendo os critérios de indicação e contraindicação da cirurgia de prótese auditiva ancorada no osso nos serviços habilitados pelo SUS, estabelecendo critérios para habilitação e financiamento dos serviços que realizarão tais procedimentos 34,35 .

No Quadro 1, podem ser visualizadas as 11 Portarias Ministeriais analisadas, discriminando os elementos encontrados em seu escopo que expressam as concepções orgânico-biológica ou socioantropológica.

\section{Discussão}

A análise do conteúdo das políticas públicas de saúde com foco na pessoa com deficiência auditiva/ surdez demonstra que não há referência às particularidades linguísticas e culturais do surdo. Conforme já apresentado no Quadro 1, todas as políticas estão pautadas na concepção orgânico-biológica da surdez, para a qual o foco não são as pessoas e suas capacidades, mas sim a correção/cura daquilo que o torna incapaz diante da maioria. Tal fato é traduzido na exclusão de uma parte da população a quem a política se destina, além de uma importante barreira comunicacional que pode afetar diretamente o seu cuidado integral em saúde.

Reconhece-se que todas essas políticas que regulamentaram a assistência às pessoas com deficiência auditiva cumpriram um papel importante no contexto em que foram criadas. A PNASA, por exemplo, permitiu um aparente avanço no aprimoramento das ações em saúde auditiva, ampliando o conjunto de ações desde a promoção à saúde até a reabilitação do aparelho auditivo, bem como a visibilidade às questões da deficiência, até então às margens das políticas mais gerais do SUS, o que levou à expansão dos serviços de reabilitação em função da previsão de financiamento específico 36 . Entretanto, a PNASA e outras políticas de saúde com foco na deficiência auditiva fundamentaram-se essencialmente na perspectiva biomédica de inclusão do surdo na sociedade ouvinte, impulsionadas pelas tecnologias e pela perspectiva de transformar o surdo em ouvinte, mediante a recuperação da audição, entre outras práticas de oralização ${ }^{8}$. São práticas de saber-poder impregnadas do modelo oralista, com foco em um público-alvo muito bem definido: aquele que não escuta, mas tem de escutar; o que não fala, mas tem de falar, assumindo um perfil excludente e discriminatório que não enxerga outras formas de se subjetivar pela surdez 17,18 .

A ciência moderna que se evidenciou no século XVII postula que todos os fenômenos naturais podem ser explicados com base na matéria em movimento e que o universo é uma máquina, tal qual os corpos humanos, concepção que deu margem ao desenvolvimento da medicina. Essa concepção, vinculada ao paradigma homem/máquina, passa a representar o surdo baseando-se em uma visão estritamente relacionada com a patologia, ou seja, com o déficit biológico. É nesse momento histórico que os surdos são excluídos da escola e do mundo do trabalho e obrigados a transitar no âmbito da 


\section{Quadro 1}

Políticas públicas do Sistema Único de Saúde (SUS) com enfoque na deficiência auditiva/surdez e os elementos encontrados em seu escopo que exprimem as diferentes concepções de surdez.

\begin{tabular}{|c|c|c|c|}
\hline Documento & Assunto/Tema & Concepção orgânico-biológica & Concepção socioantropológica \\
\hline $\begin{array}{l}\text { Portaria MS/SAS no } 116 \text {, de } 9 \text { de } \\
\text { setembro de } 199324\end{array}$ & $\begin{array}{c}\text { Inclui no Sistema de Informações } \\
\text { Ambulatoriais (SIA/SUS) a concessão } \\
\text { de órteses e próteses, entre os quais } \\
\text { a prótese auditiva externa com } \\
\text { amplificador de bolso e a prótese } \\
\text { auditiva com amplificador retro- } \\
\text { auricular. }\end{array}$ & Reabilitação do aparelho auditivo & Nada consta \\
\hline $\begin{array}{l}\text { Portaria MS/SAS no 126, de } 17 \text { de } \\
\text { setembro de } 199325\end{array}$ & $\begin{array}{c}\text { Cria os grupos e procedimentos } \\
\text { na tabela de procedimentos do } \\
\text { Sistema de Informações Hospitalares } \\
\text { (SIH/SUS), entre eles a cirurgia de } \\
\text { implante coclear. }\end{array}$ & Reabilitação do aparelho auditivo & Nada consta \\
\hline $\begin{array}{l}\text { Portaria MS/GM no } 1.278 \text {, de } 20 \\
\text { de outubro de } 199926\end{array}$ & $\begin{array}{l}\text { Estabelece critérios de indicação } \\
\text { e contraindicação da cirurgia de } \\
\text { implante coclear. }\end{array}$ & Reabilitação do aparelho auditivo & Nada consta \\
\hline $\begin{array}{l}\text { Portaria MS/SAS no 584, de } 21 \text { de } \\
\text { outubro de } 199927\end{array}$ & $\begin{array}{l}\text { Desvincula os valores da prótese } \\
\text { para implante coclear do } \\
\text { procedimento cirúrgico, bem como } \\
\text { inclui nas tabelas OPM e SIH/SUS } \\
\text { a prótese para implante coclear } \\
\text { multicanal e a prótese para implante } \\
\text { coclear monocanal para serem } \\
\text { cobradas junto do procedimento } \\
\text { cirúrgico. }\end{array}$ & Reabilitação do aparelho auditivo & Nada consta \\
\hline $\begin{array}{l}\text { Portaria MS/SAS no } 432 \text {, de } 14 \text { de } \\
\text { novembro de } 200028\end{array}$ & $\begin{array}{l}\text { Regulamenta no âmbito ambulatorial } \\
\text { o diagnóstico, a adaptação e o } \\
\text { acompanhamento de indivíduos } \\
\text { deficientes auditivos. Altera } \\
\text { procedimentos nas tabelas SUS. }\end{array}$ & $\begin{array}{c}\text { Reabilitação do aparelho } \\
\text { auditivo; treino de habilidades } \\
\text { auditivas }\end{array}$ & Nada consta \\
\hline $\begin{array}{l}\text { Portaria MS/GM no } 2.073 \text {, de } 28 \\
\text { de setembro de } 200430\end{array}$ & $\begin{array}{l}\text { Instituiu a Política Nacional de } \\
\text { Atenção à Saúde Auditiva. }\end{array}$ & $\begin{array}{l}\text { Promoção da saúde auditiva; } \\
\text { prevenção de afecções do } \\
\text { aparelho auditivo; reabilitação } \\
\text { do aparelho auditivo; reino de } \\
\text { habilidades auditivas }\end{array}$ & Nada consta \\
\hline $\begin{array}{l}\text { Portaria MS/SAS no 587, de } 7 \text { de } \\
\text { outubro de } 200431\end{array}$ & $\begin{array}{l}\text { Determinou a organização e } \\
\text { implantação das Redes Estaduais de } \\
\text { Atenção à Saúde Auditiva. }\end{array}$ & $\begin{array}{l}\text { Promoção da saúde auditiva; } \\
\text { prevenção de afecções do } \\
\text { aparelho auditivo; reabilitação } \\
\text { do aparelho auditivo; treino de } \\
\text { habilidades auditivas }\end{array}$ & Nada consta \\
\hline $\begin{array}{l}\text { Portaria MS/GM no 793, de } 24 \text { de } \\
\text { abril de } 201232\end{array}$ & $\begin{array}{l}\text { Institui a Rede de Cuidados à Pessoa } \\
\text { com Deficiência no âmbito do SUS. }\end{array}$ & $\begin{array}{c}\text { Promoção da saúde auditiva; } \\
\text { prevenção de afecções do } \\
\text { aparelho auditivo; diagnóstico } \\
\text { precoce; reabilitação do aparelho } \\
\text { auditivo; treino de habilidades } \\
\text { auditivas }\end{array}$ & Nada consta \\
\hline
\end{tabular}

(continua) 
Quadro 1 (continuação)

\begin{tabular}{|c|c|c|c|}
\hline Documento & Assunto/Tema & Concepção orgânico-biológica & Concepção socioantropológica \\
\hline $\begin{array}{l}\text { Portaria MS/SAS no } 1.328 \text {, de } 3 \\
\text { de dezembro de } 201250\end{array}$ & $\begin{array}{l}\text { Aprova as Diretrizes de Atenção à } \\
\text { Triagem Auditiva Neonatal (TAN), que } \\
\text { tem por finalidade a identificação } \\
\text { o mais precocemente possível da } \\
\text { deficiência auditiva nos neonatos e } \\
\text { lactentes. }\end{array}$ & $\begin{array}{l}\text { Diagnóstico precoce com vistas à } \\
\text { reabilitação do aparelho auditivo }\end{array}$ & Nada consta \\
\hline $\begin{array}{l}\text { Portaria MS/SCTIE no 18, de } 10 \\
\text { de junho de } 201434\end{array}$ & $\begin{array}{l}\text { Incorpora procedimentos relativos } \\
\text { à assistência hospitalar à saúde } \\
\text { auditiva (implante coclear e prótese } \\
\text { auditiva ancorada no osso) no SUS, } \\
\text { por recomendação da CONITEC. }\end{array}$ & Reabilitação do aparelho auditivo & Nada consta \\
\hline $\begin{array}{l}\text { Portaria MS/GM no } 2.776 \text {, de } 18 \\
\text { de dezembro de } 201435\end{array}$ & $\begin{array}{l}\text { Aprova diretrizes gerais, atualiza } \\
\text { os critérios para a indicação e } \\
\text { a contraindicação da cirurgia de } \\
\text { implante coclear, e estabelece } \\
\text { os critérios de indicação e } \\
\text { contraindicação da cirurgia de } \\
\text { prótese auditiva ancorada no osso. }\end{array}$ & Reabilitação do aparelho auditivo & Nada consta \\
\hline
\end{tabular}

medicina, processo que passou a ser chamado por alguns autores como "medicalização da surdez", passando a depender das habilidades e dos instrumentos do médico para curar aquilo que, na perspectiva dos ouvintes, lhe falta: a audição 37.

A Fonoaudiologia como disciplina e profissão surgiu a partir dos discursos pedagógico e médico, fruto de demandas políticas do contexto histórico, com o objetivo de superar diferenças de linguagem de determinados grupos sociais por meio de medidas de normalização e padronização da língua. Falar e ouvir eram os requisitos básicos para a produção "correta" da língua, porém, eram consideradas habilidades independentes no que se refere aos seus mecanismos de funcionamento. Nesse contexto, medicalizar a surdez foi quase automático, pois para desenvolver essas habilidades era necessário que novos recursos técnico-científicos fossem sendo adotados para subsidiar o diagnóstico e promover a audição plena e saudável, e estas lógicas ficam explícitas na análise realizada 17.

Em três décadas de existência, o SUS ainda apresenta grandes dificuldades que se refletem na assistência à saúde como um todo, e mais ainda na assistência às pessoas com deficiências 38 . No que diz respeito à deficiência auditiva/surdez, observamos a falta de atenção ao princípio da universalidade, uma vez que a política parece desconsiderar as pessoas surdas usuárias da língua de sinais, suas características culturais e linguísticas, reduzindo-as a uma incapacidade orgânico-funcional que precisa ser reparada. Mesmo questões centrais, como a presença de intérpretes de língua de sinais e a garantia de abordagem bilíngue no atendimento de habilitação e reabilitação, são ainda uma preocupação incomum nas diretrizes das políticas de saúde.

Na educação, essa realidade já é um pouco diferente, pois nos marcos legais da educação de surdos 11,12 são encontradas algumas normativas que asseguram um atendimento educacional adequado ao aluno surdo, tendo o bilinguismo como eixo norteador, visando a garantir a presença do intérprete de Libras e até mesmo de professores surdos, visto que são eles que vivenciam a experiência visual e, portanto, são capazes de contemplar essa singularidade linguística dos surdos. Além desses marcos legais, tem ainda o Plano Nacional de Educação 39 e o artigo 28 da Lei Brasileira de Inclusão 40, que discorrem sobre as diferentes modalidades de ensino que podem garantir a inclusão dos surdos 41 . 
O surdo faz parte de uma minoria linguística amparada pela legislação nacional por meio de um decreto 12 que estabelece diretrizes para a adequação do SUS, tais como orientações às famílias sobre as implicações da surdez e sobre a importância do acesso à Libras desde o nascimento, atendimento às pessoas surdas na rede de serviços do SUS por profissionais capacitados para o uso de Libras ou para a sua tradução e interpretação, e apoio à capacitação e formação de profissionais da rede de serviços do SUS para o uso de Libras, mas este decreto está longe de ser atendido na prática, já que as políticas públicas de saúde não ratificam este direito.

Algumas pesquisas pontuam fatores que comprovam a existência de barreiras e dificuldades de acesso das pessoas surdas aos sistemas de saúde, e ainda afirmam que a falta de acessibilidade comunicacional produz desconfortos e sentimentos negativos nos usuários surdos ${ }^{3}$. Dentre as principais barreiras existentes na assistência à saúde oferecida ao surdo no Brasil, foram identificadas a barreira linguística, ou seja, a dificuldade na comunicação do profissional com o usuário, e a barreira profissional, caracterizada principalmente pela falta de profissionais capacitados na Libras ou do tradutor e intérprete da Libras nas unidades e saúde 42 , o que pode implicar constrangimento e frustração. Atendimentos em Libras nos serviços de saúde são escassos, o que aumenta a vulnerabilidade dos surdos. Essas barreiras de acesso à rede de atenção à saúde encontradas pelas pessoas surdas não condizem com o que é preconizado como direito aos cidadãos e provocam iniquidades desde as ações de educação em saúde até a assistência 17,43,44. Soma-se a isso o fato de que a comunicação é um elemento fundamental nas relações paciente/profissional da saúde e precisa ter sua qualidade assegurada, pois sem ela a interação pode ficar prejudicada, comprometendo a compreensão e a adesão ao tratamento, podendo gerar riscos devido à ausência de clareza dos procedimentos de cuidados com a saúde.

Colocando-se em destaque a RCPD, vale salientar que esta é parte de uma política transversal chamada de Plano Nacional dos Direitos da Pessoa com Deficiência 45, que traz um conceito de deficiência muito mais próximo do que é conhecido como o modelo social da deficiência, o qual tem por foco as condições de interação entre a sociedade e as pessoas com limitações funcionais, visa à transformação das condições sociais mediante políticas públicas inclusivas, por meio das quais cabe à sociedade eliminar todas as barreiras físicas, programáticas e atitudinais para que as pessoas possam ter acesso aos serviços, lugares, informações e bens necessários ao seu desenvolvimento pessoal, social, educacional e profissional 46,47 . No entanto, não aparece nenhuma referência à pessoa surda usuária da Libras, e no que se refere ao cuidado às pessoas com deficiência auditiva/surdez, traz em seu instrutivo 48 apenas ações focadas na reabilitação do aparelho auditivo, sem fazer qualquer menção aos elementos da cultura surda ou à necessidade de intérprete de Libras durante os atendimentos.

Baseando-se em um diagnóstico de deficiência auditiva/surdez podem ser desencadeadas distintas intervenções que implicam a tomada de decisão por parte dos usuários, pais e profissionais envolvidos. Porém, a escassez de informações e conhecimentos acerca das diferentes concepções de surdez impossibilita a escolha 49, até mesmo porque não há oferta de outras abordagens que não sejam aquelas voltadas à reabilitação da função auditiva, visão que acabou por ser expressa em todas as políticas analisadas. Para que a RCPD possibilite de fato o acesso a todas as pessoas que compõem esse universo da deficiência auditiva, é fundamental que os profissionais tenham uma visão mais ampla sobre a saúde para além dos aspectos biológicos do corpo, e que ampliem e diversifiquem suas ações, acionando outros setores para além da saúde nos processos de produção do cuidado, o que no caso da surdez é imprescindível, sobretudo com a educação 17.

Uma característica que persiste até os dias atuais entre os fonoaudiólogos que trabalham no âmbito da surdez é a dicotomia entre as abordagens terapêuticas oralista ou bilíngue. Essa utópica ideia de que há um "melhor" método de ensino para surdos parte do pressuposto de que existe um sujeito surdo único, homogêneo, sobre o qual um mesmo método possa ser aplicado em larga escala, no entanto, o que existe é uma multiplicidade de formas de se subjetivar pela surdez que, por vezes, não se encaixa nos métodos propostos. Portanto, é preciso ressignificar os métodos e técnicas existentes, de modo que a aplicação dos recursos seja feita com base nas necessidades concretas e no modo de subjetivar de cada indivíduo 17.

Existem algumas iniciativas no setor saúde ao redor do mundo que são pautadas na concepção sócioantropológica ao trabalhar com membros das comunidades surdas, permitindo-lhes exercer maior controle sobre os determinantes de saúde. Por exemplo, na Escócia, onde alguns serviços priorizaram a contratação de profissionais de saúde mental com proficiência em língua de sinais britânica 
para o atendimento de surdos e suporte técnico a outras equipes de saúde mental da região. Nos Estados Unidos, o Centro Nacional de Pesquisa em Saúde de Surdos, em Nova Iorque, concentra seus esforços em uma população que se comunica principalmente por meio da língua de sinais americana, e conta com o envolvimento da comunidade surda como estratégia chave para o planejamento e o desenvolvimento de intervenções de promoção da saúde que sejam eficazes e apropriadas linguística e culturalmente para esta população específica 3 .

\section{Considerações finais}

A população de surdos constitui um grupo heterogêneo e inclui pessoas que têm vários graus de perda auditiva, utilizam diversos meios para se comunicar e pertencem a diferentes culturas.

Considerando-se apenas o que está pautado nas políticas públicas de saúde analisadas, é evidente o predomínio da concepção orgânico-biológica da surdez, como se o único caminho possível para essas pessoas fosse o desenvolvimento de habilidades auditivas e a oralização. A Libras, língua que legitima o surdo como sujeito de linguagem, é ignorada nessas políticas de saúde e, portanto, concluímos que há um grave problema no acesso das pessoas surdas sinalizantes não só à RCPD, mas a todo e qualquer serviço de saúde. É um monólogo, uma fala predominante que não incorpora a cultura surda.

A unilateralidade do campo da saúde na abordagem à deficiência auditiva/surdez contribui para dificultar o acesso aos serviços de saúde e às informações sobre cuidados em saúde, limitando a capacidade dos surdos usuários de língua de sinais alcançarem melhores condições de saúde para si mesmos e seus familiares. Nesse caso, a promoção da saúde e a prevenção de doenças requerem justiça social, que pode ser alcançada por meio da garantia de uma comunicação acessível e culturalmente apropriada, facilitada pelo auxílio de legendas e serviços de intérpretes 15. A ampliação da concepção de surdez adotada nas políticas públicas de saúde poderia aumentar não só o acesso ao cuidado em saúde geral, mas principalmente o protagonismo das pessoas surdas neste cuidado.

Nessa perspectiva, para além das tecnologias disponibilizadas nos serviços de saúde para a compensação do déficit auditivo, é preciso que o campo da saúde amplie a visão sobre os aspectos socioculturais do indivíduo surdo, extrapolando a questão biológica do aparelho auditivo para um ponto de vista antropológico, no qual o surdo passe a ser tratado como um sujeito que apreende o mundo pelas vivências e experiências prioritariamente visuais, capta as informações por meio da integridade sensorial que the é possível, compartilha códigos sociais e condutas políticas, e pode se expressar não só pela oralidade, mas também pelas mãos usando a língua de sinais 3,5 .

Voltando à discussão proposta por Canguilhem 6 , a cura é a reconquista de um estado de estabilidade das normas fisiológicas, no qual curar é criar para si novas normas de vida, pois em se tratando de normas humanas, estas são determinadas pelas possibilidades de agir de um organismo em situação social, pela expressão dos modos de viver socialmente adotados no meio. Sendo assim, a construção das políticas de saúde voltadas à pessoa com deficiência auditiva/surdez deve envolver todas essas singularidades, para que a oferta de serviços não atenda unicamente a uma determinada concepção, definindo padrões de normalidade que precisam ser alcançados, mas sim que objetivem garantir o acesso e a integralidade do cuidado a todas as pessoas que compõem o seguimento da deficiência auditiva. 


\section{Colaboradores}

C. Soleman contribuiu na concepção e desenho do trabalho, pesquisa, análise e interpretação de dados, escrita e é responsável por todos os aspectos do trabalho que necessitem de revisão e correção. A. Bousquat colaborou na concepção do trabalho, revisão crítica do conteúdo das informações, aprovação da versão final a ser publicada e é responsável pelo trabalho, providenciando as correções que forem necessárias.

\section{Informações adicionais}

ORCID: Carla Soleman (0000-0002-9478-4859); Aylene Bousquat (0000-0003-2701-1570).

\section{Agradecimentos}

Ao Conselho Nacional de Desenvolvimento Científico e Tecnológico (CNPq) em cooperação com o Ministério da Saúde pela chamada MS-SCTIEDECIT/CNPQ no 35/2018. A. Bousquat é bolsista de produtividade do $\mathrm{CNPq}$.

\section{Referências}

1. Bisol C, Sperb TM. Discursos sobre a surdez: deficiência, diferença, singularidade e construção de sentido. Psicol Teor Pesqui 2010; 26:7-13.

2. Bisol CA, Valentini CB. Surdez e deficiência auditiva - qual a diferença? (Objeto de Aprendizagem Incluir - UCS/FAPERGS, 2011). http://www.grupoelri.com.br/Incluir/down loads/OA_SURDEZ_Surdez_X_Def_Audit_ Texto.pdf (acessado em 28/Ago/2017).

3. Duarte SBR, Chaveiro N, Freitas AR, Barbosa MA, Porto CC, Fleck MPA Aspectos históri$\cos$ e socioculturais da população surda. Hist Ciênc Saúde-Manguinhos 2013; 20:1713-34.

4. Skliar C. Educação \& exclusão: abordagens sócio-antropológicas em educação especial. 5a Ed. Porto Alegre: Mediação; 2006.

5. Assêncio CB. Comunidade surda: notas etnográficas sobre categorias, lideranças e tensões [Dissertação de Mestrado]. São Paulo: Universidade de São Paulo; 2015.

6. Canguilhem G. O normal e o patológico. Rio de Janeiro: Editora Forense Universitária; 2009.

7. Perlin G, Strobel K. História cultural dos surdos: desafio contemporâneo. Educar em Revista 2014; (2):17-31.

8. Nóbrega JD, Andrade AB, Pontes RJS, Bosi MLM, Machado MMT. Identidade surda e intervenções em saúde na perspectiva de uma comunidade usuária de língua de sinais. Ciênc Saúde Colet 2012; 17:671-9.

9. Santana AP, Bergamo A. Cultura e identidade surdas: encruzilhada de lutas sociais e teóricas. Educação \& Sociedade 2005; 26:565-82.

10. Campos RMR. Ecos do silêncio: culturas e trajetórias de surdos em Macapá [Tese de Doutorado]. Fortaleza: Universidade Federal do Ceará; 2016.

11. Brasil. Lei no 10.436, de 24 de abril de 2002. Dispõe sobre a Língua Brasileira de Sinais Libras - e dá outras providências. Diário Oficial da União 2002; 25 abr.

12. Brasil. Decreto-Lei no 5.626, de 22 de dezembro de 2005. Regulamenta a Lei no 10.436 , de 24 de abril de 2002, que dispõe sobre a Língua Brasileira de Sinais - Libras, e o art. 18 da Lei no 10.098, de 19 de dezembro de 2000. Diário Oficial da União 2005; 23 dez.

13. Capelli JSC, De Cicco NNT, Barral J, Rumjanek VM. Educação de surdos no ensino superior. Rio de Janeiro: Editora UFRJ; 2019.

14. World Health Organization. Deafness and hearing loss. https://www.who.int/newsroom/fact-sheets/detail/deafness-and-hear ing-loss (acessado em 29/Jun/2020).

15. Barnett S, Mckee M, Smith SR, Pearson TA. Deaf sign language users, health inequities, and public health: opportunity for social justice. Prev Chronic Dis 2011; 8:A45. 
16. Instituto Brasileiro de Geografia e Estatística. Pesquisa Nacional de Saúde 2013. https:// www.ibge.gov.br/estatisticas/sociais/justicae-seguranca/9160-pesquisa-nacional-desaude.html? =\&t=o-que-e (acessado em 29/ Jun/2020).

17. Vianna NG, Andrade MGG. Ruídos e silêncios: uma análise genealógica sobre a surdez na política de saúde brasileira [Tese de Doutorado]. Campinas: Universidade Estadual de Campinas; 2018.

18. Ruivo NGV. Saúde auditiva e integralidade do cuidado: itinerário de usuários na rede de serviços [Dissertação de Mestrado]. Campinas: Universidade Estadual de Campinas; 2014.

19. Russo ICP, Pereira LD, Carvallo RMM, Anastásio ART. Encaminhamentos sobre a classificação do grau de perda auditiva em nossa realidade. Rev Soc Bras Fonoaudiol 2009; 14:287-8.

20. Goldfeld M. A criança surda: linguagem e cognição numa perspectiva sociointeracionista. 2a Ed. São Paulo: Editora Plexus; 1997.

21. Sá NRL. A educação dos surdos: a caminho do bilingüismo. Niterói: EDUFF; 1999.

22. Sá-Silva JR, Almeida CD, Guindani JF. Pesquisa documental: pistas teóricas e metodológicas. Revista Brasileira de História \& Ciências Sociais 2009; 1(1). https://periodicos.furg.br/ rbhcs/article/view/10351.

23. Departamento de Ações Programáticas Estratégicas, Secretaria de Atenção à Saúde, Ministério da Saúde. Manual de legislação em saúde da pessoa com deficiência. 2a Ed. Brasília: Editora do Ministério da Saúde; 2006.

24. Ministério da Saúde. Portaria no 116, de 9 de setembro de 1993. Inclui no Sistema de Informações Ambulatoriais do Sistema Único de Saúde - SIA/SUS a concessão de órteses e próteses. Diário Oficial da União 1993; 10 set.

25. Ministério da Saúde. Portaria no 126, de 17 de setembro de 1993. Cria na tabela de procedimentos do Sistema de Informações Hospitalares do Sistema Único de Saúde a cirurgia de implante coclear (SIH/SUS). Diário Oficial da União 1993; 18 set.

26. Ministério da Saúde. Portaria no 1.278 , de 20 de outubro de 1999. Estabelece critérios de indicação e contra-indicação da cirurgia de implante coclear. Diário Oficial da União 1999; 21 out.

27. Ministério da Saúde. Portaria no 584, de 21 de outubro de 1999. Desvincula os valores da prótese para implante coclear do procedimento cirúrgico de implantação, entre outras providências. Diário Oficial da União 1999; 22 out.

28. Ministério da Saúde. Portaria no 432, de 14 de novembro de 2000. Institui a ampliação do universo de concessão de aparelhos de amplificação sonora individuais (AASI) aos pacientes em tratamento pelo SUS e dá outras providências. Diário Oficial da União 2000; 16 nov.

29. Bevilacqua MC, Melo TM, Morettin M, Lopes AC. A avaliação de serviços em Audiologia: concepções e perspectivas. Rev Soc Bras Fonoaudiol 2009; 14:421-6.
30. Ministério da Saúde. Portaria no 2.073, de 28 de setembro de 2004. Institui a Política Nacional de Atenção à Saúde Auditiva. Diário Oficial da União 2004; 29 set.

31. Ministério da Saúde. Portaria no 587, de 7 de outubro de 2004. Determina que as Secretarias de Estado da Saúde dos estados adotem as providências necessárias à organização e implantação das Redes Estaduais de Atenção à Saúde Auditiva. Diário Oficial da União 2004; 8 out.

32. Ministério da Saúde. Portaria no 793, de 24 de abril de 2012. Institui a Rede de Cuidados à Pessoa com Deficiência no âmbito do Sistema Único de Saúde. Diário Oficial da União 2012; 25 abr.

33. Departamento de Atenção Especializada, Departamento de Ações Programáticas Estratégicas, Secretaria de Atenção à Saúde, Ministério da Saúde. Diretrizes de atenção da triagem auditiva neonatal. Brasília: Ministério da Saúde; 2012.

34. Ministério da Saúde. Portaria no 18, de 10 de junho de 2014. Torna pública a decisão de incorporar procedimentos relativos à assistência hospitalar à saúde auditiva (implante coclear e prótese auditiva ancorada no osso) no Sistema Único de Saúde - SUS. Diário Oficial da União 2014; 11 jun.

35. Brasil. Ministério da Saúde. Portaria no 2.776, de 18 de dezembro de 2014. Aprova diretrizes gerais, amplia e incorpora procedimentos para a Atenção Especializada às Pessoas com Deficiência Auditiva no Sistema Único de Saúde (SUS). 2014(b). Diário Oficial da União 2014; 19 dez.

36. Bevilacqua MC. Moretin M, Melo, TM, Amantini RCB, Martinez MANS. Contribuições para análise da política de saúde auditiva no Brasil. Rev Soc Bras Fonoaudiol 2011; 16:252-9.

37. Silva V. Educação de surdos: uma releitura da primeira escola pública para surdos em Paris e do Congresso de Milão em 1880. In: Quadros $\mathrm{RM}$, organizador. Estudos surdos I. Petrópolis: Arara Azul; 2006. p. 14-37.

38. Peroni FMA. Tecendo redes: itinerários terapêuticos de pacientes com câncer na macrorregião de Campinas-SP [Tese de Doutorado]. Campinas: Universidade Estadual de Campinas; 2013.

39. Brasil. Lei no 13.005, de 25 de junho de 2014. Aprova o Plano Nacional de Educação - PNE e dá outras providências. Diário Oficial da União 2014; 26 jun.

40. Brasil. Lei no 13.146, de 6 de julho de 2015. Institui a Lei Brasileira de Inclusão das Pessoas com Deficiência (Estatuto da Pessoa com Deficiência). Diário Oficial da União 2015; 7 jul.

41. Fernandes EAP. Políticas públicas da educação de surdos no século XXI: análise de documentos federais, estaduais e municipais [Dissertação de Mestrado]. Campinas: Universidade Estadual de Campinas; 2019.

42. Sampaio BG, Capeli JCS, Paredes HDMT, Al- 
meida MFL, Paiva RS, Alvarez AB. Assistência à saúde ao surdo no Brasil: uma revisão da literatura. In: Castro LHA, Pereira TT, Oesterreich SA, organizadores. Ciências da saúde: campo promissor em pesquisa. v. 6. Ponta Grossa: Atena Editora; 2020. p. 65-76.

43. Pinilla S, Walther S, Hofmeister A, Huwendiek S. Primary non-communicable disease prevention and communication barriers of deaf sign language users: a qualitative study. Int J Equity Health 2019; 18:71.

44. Souza EB, Souza AP, Nascimento IP, Souza APMS, Alves APP, Silva YRO, et al. O uso de Libras no serviço de saúde: uma revisão integrativa de literatura. In: Castro LHA, Pereira TT, Oesterreich SA, organizadores. Ciências da saúde: campo promissor em pesquisa. v. 6 . Ponta Grossa: Atena Editora; 2020. p. 135-44.

45. Secretaria de Direitos Humanos. Plano Nacional dos Direitos da Pessoa com Deficiência viver sem limite. Brasília: Secretaria de Direitos Humanos; 2011.
46. Sassaki RK. Inclusão: construindo uma sociedade para todos. 5a Ed. Rio de Janeiro: WVA; 2003.

47. Bampi LNA, Guilhem D, Alves ED. Modelo social: uma nova abordagem para o tema deficiência. Rev Latinoam Enferm 2010; 18:816-23.

48. Departamento de Ações Programáticas Estratégicas, Secretaria de Atenção à Saúde, Ministério da Saúde. Rede de Cuidados à Pessoa com Deficiência no âmbito do SUS: instrutivos de reabilitação auditiva, física, intelectual e visual (Centro Especializado em Reabilitação - CER e Oficinas Ortopédicas). Brasília: Ministério da Saúde; 2014

49. Teixeira GT, Silva ABP, Lima MCMP. Concepção sobre surdez na perspectiva de mães de crianças surdas. Saúde (Santa Maria) 2015; 41:93-104.

50. Ministério da Saúde. Portaria no 1.328 , de 3 de dezembro de 2012. Diário Oficial da União 2012; 4 dez. 


\section{Abstract}

Two theoretical definitions are central to the discussion on hearing impairment and deafness: the organic/biological definition and the social/anthropological definition. They differ essentially in the definition of a hypothetical boundary of normality and in the understanding of deaf individuals as a linguistic minority. This study aimed to identify how these definitions were expressed in the health policies for persons with hearing impairment/deafness in the Brazillian Unified $\mathrm{Na}$ tional Health System (SUS). A document survey was performed with a focus on health policies for hearing impairment/deafness from 1990 to 2019. The documents $(n=185)$ were identified through the Virtual Health Library and Health Legislation System, 11 of which were selected that dealt with rulings on technological implementation or practical action in care with a focus on hearing impairment/deafness (the rest dealt with specific administrative issues). The results show that all 11 policies were based on the organic/biological conception, since no reference was found to deaf individuals' linguistic and cultural specificities. The logic may hinder access by this population to the health system, especially by increasing the communication barrier. In order to meet the principles of the SUS in practice, it is essential for health policies to incorporate sociocultural aspects, respecting deaf individuals as citizens who interact with the world mainly through their visual experiences, where sign language is a central element.

Persons with Hearing Impairments; Deafness; Sign Language; Health Policy

\section{Resumen}

Dos concepciones teóricas son centrales en la discusión sobre deficiencia auditiva y sordera: la orgánico-biológica y la socio-antropológica. Ambas son fundamentalmente distintas en la definición de una hipotética frontera entre la normalidad y la concepción de los sordos como minoría lingüistica. El objetivo de este trabajo fue identificar como ambas concepciones se expresaron en las politicas de salud, dirigidas a las personas con deficiencia auditiva/sordera, en el Sistema Único de Salud (SUS). Se realizó una investigación documental centrada en las políticas de salud, dirigidas a la deficiencia auditiva/sordera de 1990 a 2019. Los documentos $(n=185)$ se identificaron a partir de la Biblioteca Virtual en Salud y del Sistema de Legislación de la Salud, de estos fueron seleccionados 11, que versaban sobre normativas de implementación tecnológica o acción práctica en la asistencia, centrándose en la deficiencia auditival sordera, los demás tenían un carácter fundamentalmente puntual. Los resultados evidencian que todas las políticas analizadas se pautaban por la concepción orgánico-biológica, puesto que no se identificó ninguna referencia a las particularidades lingüísticas y culturales del sordo. Esta lógica puede contribuir a dificultar el acceso de esta población al sistema de salud, especialmente al aumentar la barrera comunicativa. De hecho, para que se cumplan los principios doctrinarios del SUS es central que las politicas de salud incorporen aspectos socioculturales, reflejando en sus formulaciones sobre los sordos un tratamiento donde se les considere ciudadanos, que aprehenden el mundo mediante vivencias $y$ experiencias prioritariamente visuales, en el que la lengua de signos es un elemento central.

Personas con Deficiencia Auditiva; Sordera; Lengua de Signos; Politica de Salud

Recebido em 14/Jul/2020

Versão final reapresentada em 02/Dez/2020

Aprovado em 22/Dez/2020 